\title{
L'attitude des rois de prusse a l'égard des émigrés français durant la Révolution
}

\section{Thomas Höpel}

\section{(2) OpenEdition \\ 1 Journals}

\section{Édition électronique}

URL : https://journals.openedition.org/ahrf/312

DOI : $10.4000 /$ ahrf.312

ISSN : 1952-403X

Éditeur :

Armand Colin, Société des études robespierristes

\section{Édition imprimée}

Date de publication : 1 mars 2001

Pagination : 21-34

ISSN : 0003-4436

\section{Référence électronique}

Thomas Höpel, «L'attitude des rois de prusse a l'égard des émigrés français durant la Révolution », Annales historiques de la Révolution française [En ligne], 323 | janvier-mars 2001, mis en ligne le 16 mars 2006, consulté le 22 avril 2022. URL : http://journals.openedition.org/ahrf/312 ; DOI : https://doi.org/ 10.4000/ahrf.312

Ce document a été généré automatiquement le 22 avril 2022.

Tous droits réservés 


\title{
L'attitude des rois de prusse a l'égard des émigrés français durant la Révolution ${ }^{1}$
}

\author{
Thomas Höpel
}

«Malgré tous ses défauts, sur lesquels s'étendent
complaisamment ses biographes, le roi Frédéric-
Guillaume II, neveu du Grand Frédéric, a sur son
oncle, dont il ne possède pas le génie, l'avantage
d'une bonté naturelle, assez rare chez les
princes ».

Cette description de Ghislain de Diesbach dépeint le roi de Prusse comme un «brave homme de roi, toujours ouvert et généreux à l'égard des émigrés $»^{2}$. Cette attitude constante du roi de Prusse s'est opposée dans les faits à la volonté des fonctionnaires d'État et des réfugiés huguenots, hostiles aux nouveaux arrivants. Son fils, FrédéricGuillaume III, qui lui succède en 1797 , ne tarde pas à rompre avec cette ligne de conduite désuète. Diesbach fonde sa description de Frédéric-Guillaume II sur les Mémoires de certains émigrés réfugiés à la Cour de Prusse durant la Révolution, tel le vicomte de Dampmartin dont il reprend le jugement :

« Tandis que ces derniers [les ministres d'État] mettaient de l'aigreur et de la dureté dans les mesures relatives aux émigrés, le roi se plaisait à leur accorder des preuves de bonté $»^{3}$.

2 Cet exemple pose la question de savoir si la solidarité de classe affichée par le roi et son proche entourage à l'égard des émigrés a influencé durablement la politique intérieure prussienne. Cette solidarité s'est-elle du reste exprimée toujours de la même façon? L'étendue de l'immigration française en Prusse, comme l'analyse de l'attitude développée par les Hohenzollern, semblent permettre de se faire une idée assez juste de la situation. Les conditions d'intégration des émigrés dans l'armée prussienne méritent un examen tout aussi rigoureux en raison de la richesse des documents existants.

L'ampleur de l'immigration en Prusse 
3 Bien que le gouvernement prussien ait été instruit très tôt du problème de l'émigration par le biais de son envoyé extraordinaire à Paris, l'arrivée massive des émigrés ne commença qu'après la bataille de Valmy 5539émigrés au total furent enregistrés dans les possessions prussiennes, Neuchâtel exclu. Ce chiffre ne doit être en aucun cas interprété comme un instantané mais un bilan cumulé, établi sur l'ensemble de la période révolutionnaire.

4 Le tableau qui suit donne des renseignements sur l'origine et la stratification sociale des émigrés parvenus en Prusse. La sous-catégorie b permet d'apprécier par rapport au chiffre total (a) la proportion d'émigrés ayant séjourné en Prusse pendant plus de trois mois. Ce délai réglementaire était accordé généralement aux émigrés arrivés pendant l'hiver.

5 L'arrivée des émigrés débuta (si l'on néglige le cas de Neuchâtel proche de la France) en 1793-1794 dans les provinces rhénano-westphaliennes. Ce phénomène s'étendit à la Franconie en 1794 avant de toucher le centre de la Prusse en 1795. L'attraction naturelle exercée par les villes de Berlin et de Potsdam semble s'être conjuguée dans ce dernier cas avec les conséquences diplomatiques de la paix de Bâle. Cette dernière venait en effet d'accorder un statut de neutralité aux États relevant des Hohenzollern. L'année 1796 fut marquée pour sa part par l'irruption en Silésie et en Prusse méridionale d'une vague d'émigrés fuyant les offensives menées par l'armée révolutionnaire dans le sud de l'Allemagne. Le retrait des armées françaises ne provoqua le retour que d'une partie seulement de ces émigrés.

Origine et composition sociale des émigrés parvenus en Prusse ${ }^{4}$

\begin{tabular}{|c|c|c|c|c|c|c|c|c|c|c|}
\hline \multirow[t]{2}{*}{ Origine } & \multicolumn{2}{|l|}{ Total } & \multicolumn{2}{|c|}{ Noblesse } & \multicolumn{2}{|c|}{ Clergé } & \multicolumn{2}{|c|}{ Tiers État } & \multicolumn{2}{|c|}{ N.S.P. } \\
\hline & a & $b$ & a & $b$ & a & $b$ & a & $b$ & $a$ & $b$ \\
\hline Total & 5539 & 4969 & 2346 & 2106 & 1118 & 967 & 1543 & 1382 & 531 & 513 \\
\hline France & 4308 & 3978 & 1930 & 1796 & 1003 & 878 & 1105 & 1072 & 270 & 262 \\
\hline Provinces-Unies & 524 & 512 & 151 & 147 & 7 & 2 & 165 & 162 & 201 & 201 \\
\hline Pays-Bas autrich. & 268 & 239 & 101 & 88 & 72 & 64 & 79 & 73 & 16 & 14 \\
\hline Saint-Empire & 287 & 102 & 125 & 45 & 17 & 7 & 129 & 41 & 16 & 9 \\
\hline Liège & 83 & 73 & 21 & 14 & 19 & 16 & 38 & 38 & 5 & 5 \\
\hline Prusse & 32 & 32 & 5 & 5 & - & - & 19 & 19 & 8 & 8 \\
\hline Suisse & 24 & 22 & 5 & 5 & 2 & 2 & 6 & 5 & 11 & 10 \\
\hline Italie & 10 & 8 & 5 & 3 & - & - & 3 & 3 & 2 & 2 \\
\hline Angleterre & 3 & 3 & 3 & 3 & - & - & - & - & - & - \\
\hline
\end{tabular}


Nombre d'émigrés recensé au total dans les États prussiens ${ }^{5}$

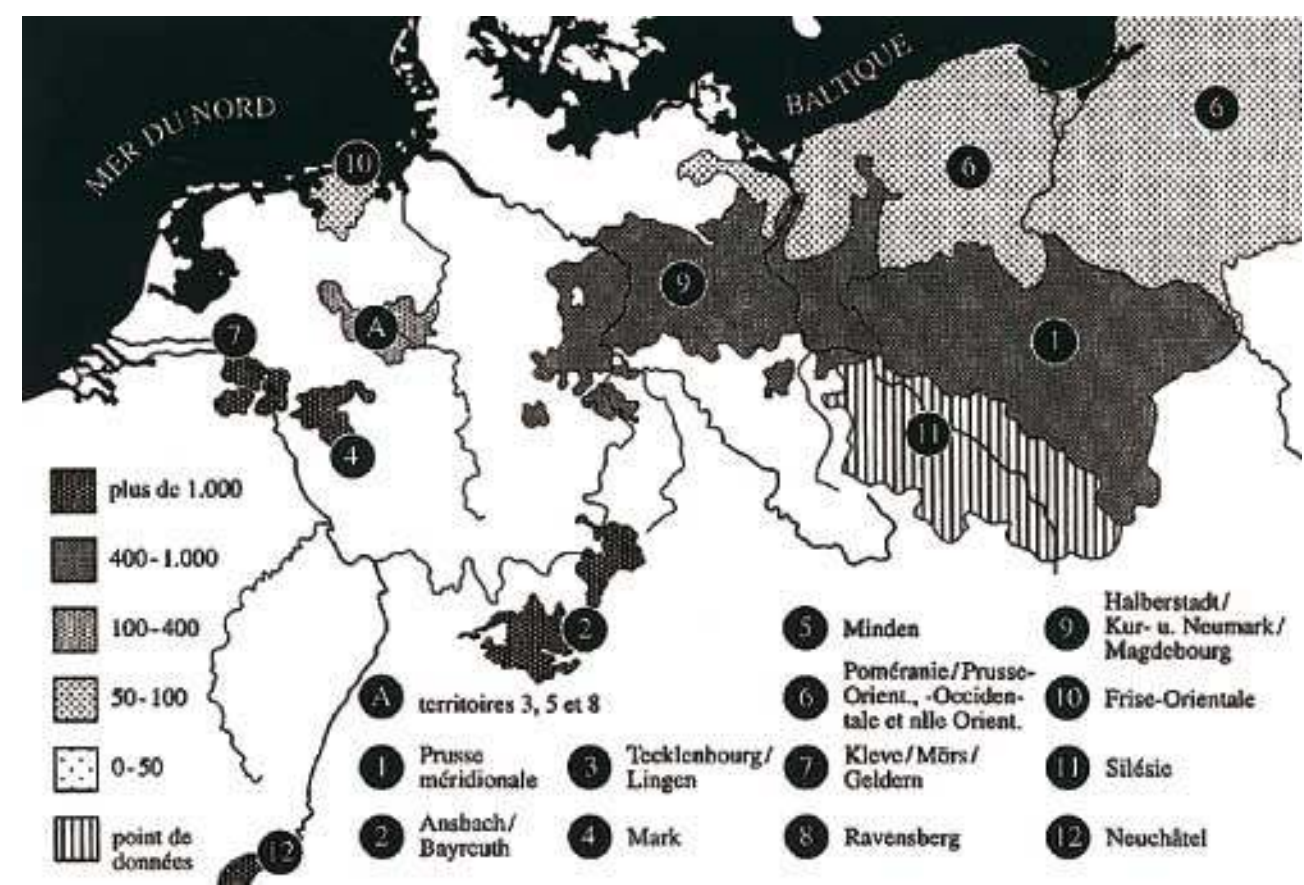

6 L'est de la Prusse ne fut guère concerné par l'immigration française. Cette dernière fut en revanche massive dans les villes de Rhénanie, Westphalie et de Franconie auxquelles il convient d'ajouter les zones de Berlin et de Varsovie en Prusse méridionale (Pologne actuelle) (voir la carte).

7 Les informations dont nous disposons sur le retour des émigrés en France sont pour certaines provinces rudimentaires en l'absence de rapports ou de tableaux généraux adressés à Berlin. On peut toutefois placer le début de ce phénomène durant l'année 1800, bien que son point culminant fût atteint en 1802 avec l'amnistie générale promulguée par Bonaparte. Les Hollandais et les Belges eurent la possibilité de rentrer dans leur patrie plus tôt en raison de la promulgation à leur endroit de lois moins restrictives. Un certain nombre de nobles et d'anciens prélats demeurèrent, quant à eux, en Prusse après 1802 pour des raisons idéologiques.

L'attitude bienveillante des rois de Prusse

8 Frédéric-Guillaume II a soutenu, comme les autres monarques européens, dès 1790, les frères du roi de France opposés au régime révolutionnaire. Il commença par donner secrètement 100000 écus au comte d'Artois par l'intermédiaire de son oncle, le prince Henri ${ }^{6}$. Après la déclaration de guerre de la France au roi de Hongrie et de Bohême, Frédéric-Guillaume II continua à soutenir les préparatifs de guerre des émigrés. Lors de la campagne d'automne 1792, l'armée prussienne prit en charge l'approvisionnement de la plus grande partie des contingents royalistes, qu'elle n'hésita pas à entretenir financièrement. Cette aide fut rompue au lendemain de la défaite de Valmy sans avoir atteint un stade inconditionnel. Les 100000 écus de 1790 n'ont jamais été en effet plus qu'un simple geste au regard des crédits alloués au même moment par la Russie ${ }^{7}$. La demande formulée par Louis XVI le 12 janvier 1792 d'une intervention militaire prussienne s'était déjà heurtée au pragmatisme de Frédéric-Guillaume II, soucieux de dédommagements financiers. Le roi de Prusse commenta cette lettre en soulignant que la question du dédommagement devait être résolue avant toute chose ${ }^{8}$. Bien que le 
plénipotentiaire de Louis XVI à Bruxelles, Breteuil, ait rassuré le roi sur ce chapitre par une lettre du premier février $1792\left({ }^{9}\right)$, le ministre d'État prussien Schulenbourg exigea une assurance écrite, signée de la main du roi de France ${ }^{10}$. Les Prussiens cherchaient à obtenir les principautés de Juliers et de Berg dont les princes régnants devaient être dédommagés en contrepartie en Alsace aux dépens de la France. Frédéric-Guillaume II sympathisa donc avec les émigrés sans subordonner pour autant à leur cause sa politique étrangère. Il ne semble jamais avoir puisé d'ailleurs dans le trésor d'État sans espérer quelques contreparties substantielles. La rupture soudaine de l'approvisionnement des émigrés peu après la bataille de Valmy révèle clairement cette inclinaison de sa politique ${ }^{11}$.

9 Frédéric-Guillaume accorda un grand nombre de permissions de séjour à des émigrés illustres comme les frères du roi de France séjournant en Westphalie à Hamm. Il fut cependant toujours en accord parfait avec la ligne politique du gouvernement d'État, hostile à l'entrée d'un grand nombre d'émigrés en Prusse. Il était aussi opposé à une ouverture de l'ensemble des territoires prussiens aux émigrés français comme l'atteste L'édit concernant le comportement des sujets pendant la guerre contre la France du 6juin $1793^{12}$. Les adoucissements postérieurs de cette ligne politique n'ont pas relevé de l'initiative propre du roi mais de celle de la Chambre domaniale et militaire de Clèves. Le souverain a témoigné plus de sentiments de solidarité dans les territoires franconiens récemment passés sous domination prussienne (d'ailleurs en bonne intelligence avec le ministre d'État gouvernant en Franconie, Hardenberg). Les émigrés nobles obtenaient ainsi plus facilement des permis de séjour à Ansbach et Bayreuth que dans le reste du domaine prussien. Divers châteaux sans occupant furent utilisés afin de loger les nouveaux arrivants, soutenus par un fonds de subvention spécial. Les autorités avaient pour principe toutefois de ne jamais sacrifier les intérêts prussiens à cette solidarité de classe. Les pratiques ayant cours en Franconie devaient se conformer (avec le nombre croissant des émigrés en Ansbach-Bayreuth) aux réglementations des autres provinces prussiennes.

Si les récits des émigrés placés sous la protection directe du roi de Prusse encensent généralement sa politique, d'autres récits plus critiques nous révèlent certains aspects moins nobles de sa personnalité. Le marquis de Bouthillier mentionne ainsi un épisode révélateur, survenu en 1793 alors que Frédéric-Guillaume était à la tête des armées coalisées. Bouthillier servait alors dans le corps de Condé soldé par les Autrichiens. Condé venait d'écrire une lettre à Frédéric-Guillaume lui exposant la situation difficile des émigrés capturés par les troupes révolutionnaires. Ces derniers risquaient en effet d'être fusillés s'ils n'étaient pas intégrés dans les conventions d'échange de prisonniers :

«Je ne puis, lui répondit ce monarque, me charger de vous comprendre (i.e. inclure) dans le cartel; des décrets lancés contre les Français par leurs compatriotes ${ }^{13}$ sont des règlements de discipline particulière, publié[s] contre des hommes qui paraissent coupables à leurs yeux. Nous nous sommes fait la loi, l'empereur d'Autriche et moi, de ne jamais nous immiscer dans les détails de leur gouvernement intérieur. Nous ne pouvons pas davantage le menacer de représailles; ce serait exposer nos propres sujets, auxquels nous nous devons avant tout, à en voir exercer de terribles contre eux. La valeur des émigrés français doit nous être un sûr garant qu'ils ne tomberont jamais vivants entre les mains de leurs ennemis $»^{14}$ 
11 Bouthillier critique avec aigreur dans le commentaire qu'il fait de cette lettre le comportement égoïste du roi de Prusse.

Le cas du marquis d'Autichamp, qui avait projeté en 1794 de créer un corps d'émigrés dans le territoire de Clèves, est comparable. Frédéric-Guillaume II, qui réprouvait ce projet, ordonna l'expulsion du marquis après l'avoir déclaré persona non grata ${ }^{15}$.

13 Frédéric-Guillaume II consentit cependant toujours à quelques exceptions comme après l'invasion des Pays-Bas autrichiens par les troupes françaises. Il accorda alors un traitement plus souple aux émigrés séjournant dans les provinces rhénanowestphaliennes, de même qu'un permis de séjour en bonne et due forme à l'ensemble des prêtres du diocèse de Metz ou émigrés présents sur le territoire prussien depuis plus d'un an et demi. Les nouveaux arrivants devaient rester dans les provinces prussiennes occidentales à condition de fournir des preuves écrites de leurs bons sentiments royalistes et d'être autonomes financièrement ${ }^{16}$. Une protestation combinée du Directoire général et du Département des Affaires étrangères, sensibles à la situation difficile de ces provinces, incita le roi à accepter une ligne politique plus dure ${ }^{17}$. Frédéric-Guillaume II approuvait d'ailleurs les mesures restrictives promulguées à l'encontre des émigrés dans les provinces centrales et particulièrement en Kurmark et à Berlin. Sa bienveillance ne dépassait donc pas les provinces occidentales de Rhénanie, Westphalie et Franconie.

14 Le roi, qui privilégiait l'intérêt de l'État, se bornait à soutenir certains émigrés choisis. Cinq d'entre eux obtinrent la charge de chambellans tandis que de petites pensions étaient allouées à d'autres. La générosité du roi s'étendait parfois à des dons de terres de dimensions modestes. Il ordonna également au gouvernement de Clèves ${ }^{18}$ de rejeter les plaintes contre les princes français concernant des dettes contractées avant leur entrée en Prusse. Frédéric-Guillaume II leur adressa d'ailleurs 200000 écus lors de leur arrivée à Hamm, fournis pour la moitié par la couronne de Russie ${ }^{19}$.

15 D'autres membres de la famille royale s'intéressèrent au sort de certains émigrés français en soutenant leur demande de permis de séjour. Quelques enfants ou adolescents intégrèrent la Cour (en tant que page) ou des instituts de cadets. Le frère de Frédéric II, le prince Henri, se distingua pour sa part en invitant à sa cour de Rheinsberg de nombreux Français qu'il connaissait de longue date ${ }^{20}$.

16 Frédéric-Guillaume II déclinait néanmoins généralement la majorité des suppliques rédigées par les émigrés afin d'obtenir une aide financière ou un asile en Prusse ${ }^{21}$. Il ne semble être intervenu qu'une fois auprès du gouvernement français, et ce en faveur d'un émigré natif des Pays-Bas autrichiens possessionné dans la principauté de Magdebourg $^{22}$. Frédéric-Guillaume II tenait donc compte des aspirations du gouvernement français dans son comportement vis-à-vis des émigrés. Il acquiesça notamment le 19mai 1797 la décision de ses ministres d'État qui avaient défendu aux émigrés le port de la Croix de Saint Louis accordée par le comte de Provence à ses favoris, suite à une plainte de l'envoyé extraordinaire français, Caillard ${ }^{23}$.

17 Son successeur, Frédéric-Guillaume III se comporta vis-à-vis des émigrés avec plus de réserve. Les émigrés, qui avaient bénéficié de la bienveillance de Frédéric-Guillaume II, le remarquèrent avec regret. Dampmartin prétend que le roi avait depuis sa prime jeunesse une aversion contre tout ce qui était français. Il avait, d'après Dampmartin, étendu ce rejet sur les émigrés : 
«L'éloignement pour leur langue s'étendit bientôt sur les Français. La Révolution ne pouvait, par les excès qui l'avaient suivie, que blesser un esprit droit et une âme honnête. De plus, une plainte assez généralement répandue disait que les Français admis dans l'intérieur de Frédéric-Guillaume II avaient contribué par leurs exemples et par leurs discours aux dépenses ainsi qu'aux faiblesses qui avaient répandu quelques nuages sur son règne. [...] Dès lors les émigrés reçus dans le monde virent la froideur succéder à l'empressement $»^{24}$.

La marquise de Nadaillac soutient que son premier décret aurait eu pour objectif d'expulser l'ensemble des émigrés résidant en Prusse. Il aurait invité simultanément le prince de Brunswick à faire la même chose dans sa principautée ${ }^{25}$.

fait, le changement à la tête de la monarchie prussienne ne semble pas avoir provoqué une telle césure. La pression prussienne exercée sur le duc de Brunswick ne résultait nullement de l'animosité du nouveau roi, mais de la pression du gouvernement français conforté par le coup d'État du 19fructidor. L'envoyé prussien à Paris, Sandoz Rollin, rapporte en effet le 7novembre 1797 que :

«Blanckenbourg est regardé ici comme le centre de toutes les conspirations contre

le Directoire, et irrite en conséquence les esprits $»^{26}$.

L'envoyé français à Berlin, Caillard, intervint le 7 décembre 1797 dans ce sens auprès du ministre de cabinet, Haugwitz. L'argument principal de Caillard était que Blanckenbourg se trouvait dans la zone de neutralité définie par le traité international du 5 août $1796^{27}$. C'était aussi l'intervention française qui incita Frédéric-Guillaume III à interdire aux émigrés le port de toute décoration d'Ancien Régime ${ }^{28}$.

Frédéric-Guillaume III à la différence de son père était opposé à toute exception à la règle. Il pensait que les décrets existants étaient suffisamment pertinents pour qu'aucune entorse n'y fût faite. Un ordre de cabinet du 6décembre 1797 nous révèle sa position au lendemain de son entrée en fonction :

«Il faudroit que des raisons bien particulières motivassent le vœu d'un émigré françois pour que je m'écartasse des ordonnances sages tendantes à éloigner de mes États des sujets onéreux \& si rarement utiles $»^{29}$.

On ne peut cependant pas parler d'une rupture profonde avec la politique de son père dans la mesure où Frédéric-Guillaume III soutenait financièrement le projet d'une colonie émigrée implantée en Prusse méridionale. Cet établissement dirigé par le chevalier de Boufflers était loin de recevoir le même suffrage de la part des ministres d'État prussiens, hostiles dès l'origine au projet ${ }^{30}$. Hardenberg continuait pourtant à entretenir au même moment certains émigrés nobles en Franconie avec le consentement du roi ${ }^{31}$.

L'attitude développée par Frédéric-Guillaume III à l'égard du comte de Provence au lendemain de son expulsion de Mitau par le tsar (1801) est à ce titre très révélatrice. Le monarque lui permit de rester temporairement avec sa suite en Prusse sous le pseudonyme du comte de l'Isle. Le prétendant français dut s'occuper personnellement du logement de la nombreuse escorte qui l'accompagna en mars 1801 à Varsovie ${ }^{32}$. Frédéric-Guillaume III lui accorda une autorisation de séjour prolongée en septembre 1801 après avoir obtenu le consentement du gouvernement français ${ }^{33}$. Le roi de Prusse ne voulait risquer aucune complication avec la France, qui pouvait déranger sa politique de neutralité en Allemagne du nord ${ }^{34}$. Il accorda par la suite à un certain nombre de parents et de courtisans du comte de Provence expatriés l'autorisation de gagner Varsovie. Les émigrés, qui sollicitaient une telle permission, devaient se soumettre à un examen approfondi. La découverte d'un nid d'espions implanté à 
Bayreuth («l'agence de Souabe») poussait en effet les autorités prussiennes à la prudence. Frédéric-Guillaume III concéda au même moment au comte de Provence une protection efficace contre les créanciers des années 1791-1792 en quête de remboursements ${ }^{35}$. Il ne prit cependant jamais le risque de le soutenir financièrement ou de l'exonérer de ses dettes. Il essaya au contraire de racheter ses créances qui atteignaient alors la somme considérable de 925000 écus $^{36}$.

Le roi de Prusse demanda au comte de Provence de s'abstenir de toute action politique susceptible de compromettre ses relations avec la France en contrepartie de son hospitalité. Les autorités prussiennes remarquèrent rapidement la mauvaise volonté du comte de Provence. Ce dernier se vit refuser à ce titre un lieu d'hébergement plus occidental. Frédéric-Guillaume III affirme notamment dans une de ses lettres que

«le comte de Provence court après le fantôme d'une contre-révolution. Notre relation momentanée avec la France, le calme en Europe, notre propre salut demandent de contrecarrer de tels projets. On ne peut avoir aucune confiance ${ }^{37}$ dans le comte ou son environnement. On ne peut pas attendre non plus de lui la discrétion voulue $»^{38}$.

Frédéric-Guillaume III mit en garde le comte de Provence contre ces dangers au mois de juillet 1804 alors que ce dernier se préparait à partir de Varsovie pour rencontrer plusieurs membres de sa famille. Le roi mit la poursuite du séjour du comte à Varsovie dans la balance afin de minimiser les risques de conspiration. Le séjour du frère de Louis XVI en Pologne était devenu à cette date par sa notoriété la pierre d'achoppement des relations franco-prussiennes. Une prétendue tentative d'empoisonnement eut lieu contre le comte de Provence et sa suite peu avant son départ. Le roi de Prusse comme ses ministres d'État ou ses fonctionnaires de Varsovie ne croyaient guère en la solidité de cet attentat exécuté avec trop de dilettantisme. Le conseiller secret de cabinet Beyme écrivit à Hardenberg le 31 juin 1804 :

« D'ailleurs, cette dénonciation semble à Sa Majesté dénuée de tout fondement. Une enquête criminelle est cependant nécessaire. Il faudra vérifier si cette dénonciation ne se fonde que sur une intrigue et en punir convenablement l'auteur, surtout s'il s'agit de Coulon $»^{39}$.

26 L'enquête judiciaire portait à croire que ce complot avait été mis en scène par des membres de la suite du comte afin d'alimenter la propagande anti-révolutionnaire. Les émigrés ne tardèrent pas à publier un article dans le Courrier de Londres dans ce sens :

«Nous donnons le défi à tous les éditeurs de papiers anglois, et à tous les journalistes de Buonaparte de contester l'authenticité des détails, que nous publions et si les papiers françois osent entreprendre de justifier M. Buonaparte de ce nouveau crime, nous nous chargerons de démontrer, qui en est le véritable auteur $»^{40}$.

Cette version orientée entache encore de nombreuses publications consacrées à l'histoire des émigrés en raison de l'enterrement du dossier par les autorités prussiennes ${ }^{41}$. Les enquêteurs n'ayant trouvé aucune preuve probante attribuèrent la paternité de l'attentat à un domestique du comte de Provence. Le département des Affaires étrangères, soucieux d'apaiser les tensions causées par cette affaire, inséra un article dans plusieurs journaux innocentant l'agent commercial français montré du doigt par les royalistes. L'enquête finit par inquiéter un baron de l'entourage immédiat du comte de Provence avant d'être enterrée à la suite d'une décision du gouvernement prussien. Hardenberg déclara le 5 février 1805 au grand chancelier Goldbeck qu'on ne pouvait poursuivre l'enquête 
«sans compromettre plusieurs personnes de l'entourage du comte de l'Isle. Le départ de celui-ci la rendrait du reste par trop difficile $»^{42}$. Son soutien se subordonna toujours aux exigences du budget de l'État et au maintien de bonnes relations franco-prussiennes. Frédéric-Guillaume II avait réduit de la même façon son soutien financier après 1792 en raison de ses aspirations politiques comme de la situation précaire de ses finances ${ }^{46}$. De même après la paix de Bâle, FrédéricGuillaume II s'efforça de ne pas compliquer les relations franco-prussiennes par un soutien trop manifeste des émigrés.

L'insertion des émigrés dans l'armée prussienne

33 La noblesse française a sacralisé sous l'Ancien Régime son rôle d'élite à travers sa contribution militaire qualifiée "d'impôt du sang ${ }^{47}$. Ce facteur d'identité a amené beaucoup d'émigrés à chercher à entrer dans l'armée prussienne à un moment où cette dernière s'auréolait d'une réputation internationale. Les Prussiens, dépassés par les événements, se montrèrent très réservés envers ces nombreuses demandes. Le principe de la raison d'État poussait à protéger les officiers issus de familles prussiennes, dont on attendait un fort patriotisme. La politique des rois de Prusse depuis FrédéricGuillaume Ier visait à créer un corps d'officiers recruté dans le royaume même de façon à aboutir à une symbiose entre l'armée et la noblesse prussienne ${ }^{48}$. Il n'y avait que deux sortes d'exceptions à ce principe fondamental, en cas de compétences extraordinaires, ou d'une intervention du roi en faveur d'un impétrant (42 cas au total). On acceptait dans le deuxième cas de jeunes émigrés descendant de familles prestigieuses. Ces derniers commençaient leur service dans des écoles militaires ou avec le grade d'officier subalterne en cas d'intégration directe (37cas). Frédéric-Guillaume III exigeait qu'ils apprissent très rapidement l'allemand ${ }^{49}$ afin de faciliter leur socialisation rapide 
dans l'armée prussienne, que le roi considérait comme la première étape de leur assimilation politique. Les documents d'archives révèlent que seize émigrés intégrés ainsi dans l'armée prussienne y demeurèrent après l'amnistie de 1802.

Le cas du comte de Rézicourt est particulièrement représentatif de ce système d'assimilation. Ce noble lorrain, qui avait obtenu en 1798 la permission d'acheter un domaine rural à Ansbach en Franconie, était devenu par là même sujet du roi de Prusse. Il chercha à placer dès 1801 son fils de douze ans dans l'École des cadets de Berlin avec l'appui du ministre d'État Hardenberg. Le commandant en chef de l'école s'y opposa par une lettre dans laquelle il affirme qu'

« il existe un ordre explicite qui permet seulement à des fils de nobles prussiens nés en Prusse d'être éduqués dans cet institut $»^{50}$.

Hardenberg conseilla alors au comte de Rézicourt de s'adresser directement au roi en lui présentant son fils lors de son prochain séjour en Franconie. L'acquisition de son domaine d'Ansbach était donc à cette date trop récente pour permettre à son fils d'intégrer le corps d'officiers sur lequel la société prussienne se fondait ${ }^{51}$.

Conclusion

Les souverains prussiens ne furent jamais obligés de prouver leur patriotisme par une attitude politique rigoriste, conforme à la raison d'État alors qu'elle animait leurs ministres ou leurs hauts fonctionnaires. Ils représentaient l'union des États prussiens par leur personne. Quand ils montrèrent plus de solidarité pour les émigrés que les hauts fonctionnaires, ce n'était donc pas parce qu'ils étaient de mauvais patriotes. Cela montre plutôt qu'ils étaient en tant que rois de Prusse aussi des représentants du principe monarchique. Leur attitude à l'égard des émigrés a constamment oscillé en fonction de ces deux facteurs identitaires. En cas de conflit d'intérêts, ce fut toujours la raison d'État qui eut le dernier mot et non la solidarité de classe.

L'importance de la raison d'État se montra clairement dans le cas de l'insertion des émigrés dans l'armée prussienne qui était un instrument fondamental pour l'intégration de la monarchie prussienne. Les rois ne permirent l'insertion des émigrés à ce corps d'élite que s'ils étaient soit utiles par leurs hautes compétences de la technique militaire soit assez jeunes pour pouvoir adopter les sentiments du patriotisme prussien.

\section{NOTES}

1.Mes remerciements vont à Pierre-Vincent Claverie (Université de Paris I) pour sa lecture critique et attentive de la version française.

2.Ghislain DE DIESBACH, Histoire de l'Émigration 1789-1814, Paris, 1975, pp. 336-338.

3.Anne-Henri DAMPMARTIN, Mémoires sur divers événements de la Révolution et de l'Émigration, Paris, 1825, t.II, p. 236. Un jugement comparable sur Frédéric-Guillaume II transparaît du témoignage fourni en 1792 par le vicomte de Caraman, alors colonel dans l'armée prusienne (cf. "Mémoires du Duc de Caraman, Revue contemporaine, t. X, 1853, p. 379). Les mêmes preuves de bonté à l'égard des émigrés ressortent des 
Mémoires de la marquise de Nadaillac et du duc Des Cars: Mémoires de la marquise de Nadaillac, duchesse d'Escars suivis des mémoires inédits du duc d'Escars, publiés par son arrière-petit-fils le colonel marquis de Nadaillac, Paris, 1912, p. 242 ; Mémoires du duc Des Cars, publiés par son neveu le duc Des Cars, Paris, 1890.

4.Les chiffres reposent sur une banque de données établie par l'auteur. Elle rassemble les noms de tous les émigrés enregistrés dans les documents bureaucratiques prussiens déposés dans les archives à Berlin, Munster, Dusseldorf, Bayreuth et Ansbach.

5. Ibid.

6.Geheimes Staatsarchiv Preußischer Kulturbesitz Berlin (GStA) PK, I. HA, Rep. 96, Geheimes Zivilkabinett, $\mathrm{n}^{\circ} 148 \mathrm{~K} 2$.

7.Catherine II devança dans son soutien précoce le tsar Paul I qui constitua une rente en faveur du comte de Provence lui permettant d'entretenir une vaste suite à Mitau puis Varsovie. Voir René DE CASTRIES, Les Émigrés, Paris, 1962, pp. 74, 87, 114 ; Wilhelm BRINGMANN, Louis XVIII. von Frankreich im Exil. Blankenburg 1796-1798, Frankfurt/Main 1995, p. 261.

8.GStA PK, I. HA, Rep. 11, Frankreich, n ${ }^{\circ} 89$, fasc. 298: Secretissima sur les affaires de France 1792, Schulenburg à Frédéric-Guillaume II, Berlin, 13 janvier 1792.

9.GStA PK, I. HA, Rep. 11, Frankreich, n 89 , fasc. 298: Secretissima sur les affaires de France 1792, Breteuil à Schulenburg, Bruxelles, 1er février 1792.

10.GStA PK, I. HA, Rep. 11, Frankreich, $n^{\circ} 89$, fasc. 298: Secretissima sur les affaires de France 1792, Schulenburg à Breteuil, Berlin, 13 février 1792.

11.GStA PK, I. HA, Rep. 11, n 91 a, Französische Emigranten, Gen. fasc. 1, Vol. 1 (M): Note de Lucchesini, 7 novembre 1792.

12.Titre original: "Edict das Verhalten der Königlichen Unterthanen bey dem gegenwärtigen Kriege mit Frankreich betreffend. Berlin 6. Juny 1793: GStA PK, I. HA, Rep. 11, n 91 a, Französische Emigranten, Gen. fasc. 1, Vol. 1 (M).

13.Ms.: "compatriotes émigrés (à la suite d'une confusion).

14.André DE MARICOURT, "L'armée de Condé. Le marquis de Bouthillier et ses Mémoires, Revue des questions historiques, 3e série, t. VI, 1925, pp. 354-355.

15.Rescrit au Gouvernement de Clèves et au Chambre domaniale et militaire de clèves, Berlin, 23août 1794: GStA PK, I. HA, Rep. 11, nº 91a, Französische Emigranten, Gen. fasc. 4.

16.Ordre de cabinet, camp proche d'Oppalin, 14 juillet 1794: GStA PK, I. HA, Rep. 11, $\mathrm{n}^{\circ}$ 91 a, Französische Emigranten, Gen. fasc. 1, vol. 2 (M).

17.Ordre de cabinet au ministère d'État, camp de Wola, 5 septembre 1794: GStA PK, I. HA, Rep.11, n 91 a, Französische Emigranten, Gen. fasc. 1, vol. 2 (M).

18.Les gouvernements étaient dans l'ancienne Prusse des cours de justice provinciales. 19. René DE CASTRIES, op. cit., Paris, 1962, p. 114.

20.Fernand BALDENSPERGER, "L'émigration de chevalier de Boufflers, Revue de Paris, 1912, pp.791-801.

21.On trouve plus de cent suppliques de ce genre dans les seuls fascicules 1 à 44 de la série des documents GStA PK, I. HA, Rep.11, $n^{\circ}$ 91b, Französische Emigranten - Unbestimmter Aufenthalt, Spez. (M). La plupart restèrent lettres mortes en raison de l'hostilité du roi. 22.GStA PK, I. HA, Rep. 11, n 91, Frankreich - Privata, fasc. 36, Acta betr. die von dem Grafen von Merode-Westerloo nachgesuchte Königliche Verwendung bey Frankreich, wegen seiner Reichsbaronie Petersheim.

23.Ordre de cabinet, Potsdam, 19 mai 1797: GStA PK, I. HA, Rep.11, n 91a, Französische Emigranten, Gen. fasc. 7. 
24.Anne Henri DAMPMARTIN, op. cit., Paris, 1825, t.II, p. 280.

25.Mémoires de la Marquise de Nadaillac (...), op. cit., p. 111.

26.GStA PK, Rep. 11, $n^{\circ}$ 93h, Frankreich -Allianzen, $n^{\circ} 3$, fol. 10.

27.GStA PK, Rep.11, n93h, Frankreich - Allianzen, $n^{\circ} 3$, Bl. 10-28. Voir aussi Wilhelm BRINGMANN, Louis XVIII. von Frankreich im Exil. Blankenburg 1796-1798, Frankfurt/Main 1995, pp. 239-241.

28. Voir Département des Affaires étrangères au Collège supérieur de guerre, Berlin, 20 mars 1798: GStA PK, I. HA, Rep. 11, n 91a, Französische Emigranten, Gen. fasc. 7.

29.GStA PK, 1. HA, Rep.11, nº 91b, Französische Emigranten - Unbestimmter Aufenthalt, fasc.spéc. 50 (M).

30.GStA PK, I. HA, Rep.11, n 91b, Französische Emigranten in Südpreußen, fasc. spéc. 66 (M).

31.Voir le rapport de HARDENBERG "Die Unterstützung der französi. Emigranten aus den könig. Kassen betr., Ansbach, 23 décembre 1797: GStA PK, I. HA, Rep. 96 A, n 19, Aufenthalt französischer Emigranten in Preußen. 1797-1804, Bl. 1 ; et la lettre de Hardenberg au Département des Affaires étrangères, Ansbach, 6 juillet 1799: GStA PK, I. HA, Rep.11, n 91b, Französische Emigranten in Ostpreußen und Neuostpreußen, fasc. spéc. 7.

32. Ordre de cabinet à Alvensleben et Schroetter, Berlin, 21 février 1801: GStA PK, Rep. 11, n93h, Frankreich - Allianzen, n 3, Bl. 95.

33. Ordre de cabinet, Potsdam, 29 septembre 1801: GStA PK. HA, Rep. 11, n 93h, Frankreich - Allianzen, $n^{\circ}$ 3., Bl. 144.

34.Concernant la politique de neutralité de la Prusse de 1795 à 1805 voir Philip DWYER, “The Politics of Prussian Neutrality 1795-1805”, German History, t. 12 (fasc. 3), pp. 351-373.

35. Ordre de cabinet, Potsdam, 2 octobre 1802: GStA PK. HA, Rep. 11, n 93h, Frankreich - Korrespondenz mit französischen Herrschern, n 5, Bl. 52.

36.Frédéric-Guillaume II avait allégé la dette des princes français de 530583 écus ( $c f$. GStA PK, I. HA, Rep. 96 A, nº 9 B b 5).

37.Ms: "ne peut pas avoir (sic).

38. Lettre du conseiller secret de cabinet Beyme, Charlottenbourg, 21 août 1802: GStA PK, Rep.11, n 93h, Frankreich - Allianzen, n³, Bl. 181-182. Citation originale: "Unser gegenwärtiges Verhältniß mit Franckreich, die Ruhe von Europa, unsre eigne Wohlfahrt erfordern solchen Absichten geradezu entgegen zu arbeiten. Vertrauen kann mann ohnehin in den Grafen und seine Umgebung nicht setzen. Discretion kann mann gar nicht erwarten. 39. Citation originale: "Uebrigens scheint auch Sr. May. die gantze Anzeige bloß aus der Luft gegriffen, dennoch es aber nöthig zu sein, eine förmliche Criminal-Untersuchung zu eröfnen, damit wenn die Anzeige auf eine bloße Intrigue beruhen sollte dieses ausgemittelt und der Urheber derselben, besonders aber der Coulon zur gebührenden Strafe gezogen werde: GStA PK, I. HA, Rep. 96A, Geheimes Zivilkabinett, nº 9 A4 ; Schriftwechsel der Kabinettsminister Alvensleben und Haugwitz mit Beyme in vermischten Angelegenheiten des Auswärtigen Departements, 1799-1804.

40.Rapport du préfet Meyer rédigé à Varsovie le 20 septembre 1804 (copie): GStA PK, Rep. 11, n 93h, Frankreich - Korrespondenz mit französischen Herrschern, $n^{\circ} 4$, betr. die angeblich beabsichtigte Vergiftung des Grafen de L'Isle und dessen Familie und den deshalb denunciirten Coulon zu Warschau.

41.Voir René DE CASTRIES, op. cit., pp. 348-349 ; René DE CASTRIES, La vie quotidienne des émigrés, Paris, 1966, pp. 287-288 ; Ghislain DE DIESBACH, op. cit., p. 348 ; Évelyne LEVER, 
Louis XVIII, Paris, 1988, p. 281. Philip MANSEL donne un jugement plus nuancé (cf. Louis XVIII, Gloucestershire, 1999, p. 81).

42. Hardenberg à Goldbeck, Berlin, 4 février 1805: GStA PK, Rep. 11, nº 93h, Frankreich Korrespondenz mit französischen Herrschern, $n^{\circ} 4$. betr. die angeblich beabsichtigte Vergiftung des Grafen de L'Isle und dessen Familie und den deshalb denunciirten Coulon zu Warschau. Citation originale: “Ohne mehrere Personen des Gefolges des Grafen von Lille mit hinein zu ziehen, dieses aber bey ihrer nunmehrigen Entfernung mit vielen Weitläuftigkeiten verknüpft ist [...].

43.Le comte de Provence et le comte d'Artois rédigèrent alors au nom de l'ensemble de leur famille une protestation contre le couronnement de Napoléon afin de contester la légitimité de son sacre en rappelant leurs prétentions sur la couronne de France. Cette déclaration fut rédigée sur mer en raison de la neutralité affichée par les différentes monarchies européennes ( $c f$. René DE CASTRIES, op. cit., pp.351-353 ; Jacques GODECHOT, La Contre-Révolution 1789-1804, Paris, 1961, pp. 404-405).

44. Rescrit à von Tarrach, Berlin, 5 octobre 1804: GStA PK, Rep. 11, n 93h, Frankreich Allianzen, $\mathrm{n}^{\circ}$ 3, Bl. 215-216.

45.Ordre de cabinet adressé à Haugwitz le 27 juin 1801 de Charlottenbourg: GStA PK, I. HA, Rep.11, n 91b, Französische Emigranten in Ansbach-Bayreuth, fasc. spéc. 46 (M).

46.Voir sur ce dernier point Wolfgang NEUGEBAUER, “Zur neueren Deutung der preußischen Verwaltung im 17. und 18. Jahrhundert. Eine Studie in vergleichender Sicht, Jahrbuch für die Geschichte Mittel- und Ostdeutschlands, t. XXVI, 1977, p. 99.

47. Ce thème devint un sujet de débat en France à la suite de la publication de La noblesse commerçante de l'abbé Coyer en 1756. Le chevalier d'Arcq y répondit la même année par un pamphlet intitulé La noblesse militaire ou le patriote français par lequel il entendait défendre la vocation militaire de l'aristocratie française (cf. Pierre SERNA, "Der Adlige, Der Mensch der Aufklärung, éd. M. Vovelle, Frankfurt/Main-New York, 1996, pp. 74-76 ; Pierre GOUBERT, Daniel ROCHE, Les Français et l'Ancien Régime. La société et l'État, Paris, 1984, p. 136).

48. Concernant l'aristocratisation du corps des officiers prussiens au XVIIIe siècle voir Karl DEMETER, Das deutsche Offizierskorps in Gesellschaft und Staat 1650-1945, Frankfurt/ Main, 1965, pp. 2-5 ; Otto BÜSCH, Militärsystem und Sozialleben im alten Preußen 1713-1807, Frankfurt/Main-Berlin-Wien, 1981, spécialement le chapitre "Adel und Offiziersdienst", pp. 79-84.

49.Le roi répondit favorablement, selon le ministre d'État Lucchesini, à la supplique du marquis de Boyer d'Eguilles en faveur de l'incorporation de ses fils à la condition d'apprendre l'allemand avant d'être cantonnés en Pologne dans de nouveaux régiments prussiens ( $c f$. Supplique du marquis de Boyer d'Eguilles, Düsseldorf, 21 mars 1793: GStA PK, I. HA, Rep. 96, Geheimes Zivilkabinett, n 148 K1 [Die französischen Emigranten 1790-1797]). Dans le cas d'Antoine Charles de Tabouillot, Frédéric-Guillaume II promit l'intégration dans un régiment de hussards après une amélioration sensible de son niveau de langue ( $c f$. GStA PK, I. HA, Rep. 11, n 91 b, Französische Emigranten in der Kur - und Neumark, fasc.spéc. 27).

50.Lingelsheim à Hardenberg, Berlin, 8 janvier 1802: GStA PK, II. HA, Generaldirektorium - Ansbach-Bayreuth, Abt. IV, Militärsachen, ${ }^{\circ} 47$. Citation originale: "Da der ausdrücklichen Vorschrift gemäß nur im Lande gebohrnen Söhnen preußischer Vasallen, das Beneficium der in dieser Anstalt zu genießenden Erziehung theilhaftig werden solle. 
51.GStA PK, II. HA, Generaldirektorium - Ansbach-Bayreuth, Abt. IV, Militärsachen, $\mathrm{n}^{\circ}$ 47.

\section{RÉSUMÉS}

Entre 1789 et 1806, plus de 5500 émigrés furent enregistrés dans les États prussiens. Le but de l'article est de savoir si la solidarité de classe affichée par le roi et son proche entourage à l'égard des émigrés a influencé durablement la politique intérieure prussienne. Les rois prussiens montrèrent plus de solidarité de classe vis-à-vis des émigrés que les hauts fonctionnaires. Néanmoins, la raison d'État était aussi pour eux prédominante et les empêcha de recevoir beaucoup d'émigrés en Prusse. L'importance de la raison d'État se montra clairement dans le cas de l'insertion des émigrés dans l'armée prussienne qui était un instrument fondamental pour l'intégration de la monarchie prussienne. Les rois ne permirent l'insertion des émigrés à ce corps d'élite que s'ils étaient soit utiles par leurs hautes compétences de la technique militaire, soit assez jeunes pour pouvoir adopter des sentiments d'un patriotisme prussien.

The attitude of the Prussian kings towards the French émigrés during the Revolution.

Between 1789 and 1806, more than 5500 émigrés were registered in the Prussian territories. The objective of the article is to show the extent to which class solidarity influenced the politics of the King and the royal family vis-à-vis the émigrés. Was this principle of class solidarity only relevant in specific cases or was it dominant? The Prussian monarchs showed a higher solidarity with the émigrés of the French revolution than the Prussian government officials. However, for the Prussian kings the welfare of the State was of highest importance and prevented them from receiving a lot of émigrés of the French revolution in the Prussian territories. The role of Prussian patriotism and of the "raison d'état» is to be seen obviously in the attitude of the Prussian kings concerning the admission of French émigrés in the Prussian army. Such admissions were widely refused. The army was together with the bureaucracy the most important institution for the integration of ancient Prussia. Prussian officers had to be also Prussian patriots which was not at all the case for French émigrés.

INDEX

Mots-clés : émigrés, raison d'État, solidarité de classe, patriotisme prussien

\section{AUTEUR}

THOMAS HÖPEL

Université de Leipzig 